\title{
BMJ Open Standard maintenance therapy versus local consolidative radiation therapy and standard maintenance therapy in 1-5 sites of oligometastatic non-small cell lung cancer: a study protocol of phase III randomised controlled trial
}

\author{
Anil Tibdewal (1) , ${ }^{1}$ Jai Prakash Agarwal, ${ }^{1}$ Shashank Srinivasan, ${ }^{1}$ \\ Naveen Mummudi, ${ }^{1}$ Vanita Noronha, ${ }^{2}$ Kumar Prabhash, ${ }^{2}$ Vijay Patil, ${ }^{2}$ \\ Nilendu Purandare, ${ }^{3}$ Amit Janu, ${ }^{4}$ Sadhna Kannan ${ }^{5}$
}

To cite: Tibdewal A, Agarwal JP, Srinivasan S, et al. Standard maintenance therapy versus local consolidative radiation therapy and standard maintenance therapy in $1-5$ sites of oligometastatic nonsmall cell lung cancer: a study protocol of phase III randomised controlled trial. BMJ Open 2021;11:e043628. doi:10.1136/ bmjopen-2020-043628

- Prepublication history and supplemental material for this paper is available online. To view these files, please visit the journal online (http://dx.doi. org/10.1136/bmjopen-2020043628).

Received 10 August 2020 Revised 07 December 2020 Accepted 03 February 2021

Check for updates

(C) Author(s) (or their employer(s)) 2021. Re-use permitted under CC BY-NC. No commercial re-use. See rights and permissions. Published by BMJ.

For numbered affiliations see end of article.

Correspondence to

Dr Anil Tibdewal;

aniltibdewal@gmail.com

\section{ABSTRACT}

Introduction Two-phase II randomised studies have shown a significant benefit of local consolidation therapy in oligometastatic non-small cell lung cancer (NSCLC). This phase III randomised controlled trial (RCT) will evaluate the efficacy of local consolidation radiation therapy (RT) in oligometastases (OM) NSCLC after completion of initial systemic therapy.

Methods and analysis This is a single-centre phase III RCT of OM NSCLC patients. One hundred and ninety patients will undergo 1:1 randomisation to either standard maintenance therapy (control arm) or local consolidation RT and standard maintenance therapy (experimental arm). Patients will be stratified into the number of $0 \mathrm{M}$ sites (1-2 vs 3-5), nodal metastases (N0-N1 vs N2-N3) and presence or absence of brain metastases. Stereotactic body radiation therapy to all the oligometastatic sites and definitive RT to primary disease will be given in the experimental arm. The primary endpoint is overall survival and secondary endpoints include progression-free survival, local control of OM sites, new distant metastases free survival, objective response rate, toxicity and quality of life. Translation endpoint include circulating tumour cells and radiomics using texture analysis.

Ethics and dissemination All patients will be provided with a written informed consent form which needs to be signed before randomisation. The study is approved by the institutional ethics committee-II (project number 3445) and registered with Clinical Trials Registry_-India, dated 21 April 2020.

Trial registration number CTRI/2020/04/024761; PreResults.

\section{INTRODUCTION}

Systemic therapy is the standard of care for patients with metastatic non-small cell lung cancer (NSCLC). Tyrosine kinase inhibitors (TKI) have significantly improved survival outcomes for patients with an actionable
Strengths and limitations of this study

Used consensus definition for number of oligometastases sites.

- Randomisation after initial systemic chemotherapy if no progression.

- Practical eligibility criteria for timely recruitment.

- Stereotactic body radiation therapy to all oligometastatic sites.

- Translational endpoints of circulating tumour cells and radiomics analysis.

oncogene mutation like epidermal growth factor receptor (EGFR) or anaplastic lymphoma kinase (ALK) ${ }^{1-4}$ In patients with programmed death/ligand receptor expression, immune checkpoint inhibitors also improves outcomes compared with systemic therapy alone. ${ }^{56}$ Patients who do not have oncogene mutations and are not eligible for immunotherapy have a worse prognosis with median overall survival (OS) ranging from 10 to 13 months as compared with median OS of 18-26 months for patients treated with TKI or immunotherapy. ${ }^{46}$

Metastatic NSCLC with limited sites of metastases referred to as oligometastases $(\mathrm{OM})$ has shown better prognosis than those with widespread metastases. ${ }^{78}$ The $\mathrm{OM}$ state was proposed as an intermediate stage of cancer with a spread between localised disease and widespread metastases. ${ }^{9} 10$ The significance of the OM paradigm is that selected patients could be cured with radical local therapies. ${ }^{11}$ There has been much debate as to the definition of oligometastatic disease 
in NSCLC. Recently, the European consensus definition for synchronous oligometastatic NSCLC was published. These include patients with a maximum of five metastatic lesions involving a maximum of three organs and all can be treated with radical local ablative therapy. ${ }^{12} 13$

The question remains why oligometastatic disease should behave differently than widespread metastatic disease. Patterns of failure analyses from limited metastatic NSCLC suggest that disease progression most often occurs at sites of existing disease at baseline rather than at new sites. ${ }^{14-16}$ Hence, aggressive treatment of limited metastatic sites could potentially remove the dominant disease that could seed other sites in the future. Various retrospective studies have proven the role of definitive local therapy in oligometastases. ${ }^{17} 18$ Two-phase II studies performed by Gomez et $a l^{19}$ and Iyengar et $a l^{20}$ showed that local consolidative therapy in addition to systemic therapy has a role in oligometastatic NSCLC. Gomez et $a l^{19}$ randomised 1-3 sites of OM NSCLC patients to local consolidative therapy with or without maintenance therapy or to maintenance treatment alone. They showed a significant median progression-free survival (PFS) benefit in favour of local consolidative therapy (11.9 months vs 3.9 months, $p=0.005$ ). Long-term results also showed an OS benefit of 41 months versus 17 months. ${ }^{19} 21$ Iyengar $e t a t^{20}$ randomised 29 patients to maintenance chemotherapy (CT) alone versus stereotactic ablative radiotherapy (SABR) followed by maintenance CT. As opposed to the study by Gomez et $a l^{19}$ they enrolled patients with negative EGFR/ALK mutations and up to five metastatic sites. They showed a significant improvement in PFS with SABR (9.7 months vs 3.5 months, $\mathrm{p}=0.01$ ). The SINDAS trial is the only phase III randomised trial with results presented at the recent American Society of Clinical Oncology (ASCO) meeting. The study randomised patient with EGFR mutation and $\leq 5 \mathrm{OM}$ sites to either TKI alone or stereotactic body radiation therapy (SBRT) plus TKIs. The study showed a significant median PFS (20.2 vs 12.5 months, $\mathrm{p}<0.001)$ and $\mathrm{OS}(25.5$ vs 17.4 months, $\mathrm{p}<0.001)$, respectively. ${ }^{22}$
Although there has been promising data for the addition of local consolidative therapy to standard systemic therapy for oligometastatic NSCLC, these studies remain non-definitive as they included small patient numbers. The only reported phase III randomised controlled trial (RCT) has exclusively selected patients with EGFR mutation. ${ }^{22}$ These patients have a different natural history and outcomes for those who do not have EGFR mutations. ${ }^{23}$ Hence, we initiated a phase III RCT to ascertain the role of addition of local consolidative radiation therapy (LCRT) to standard maintenance therapy (SMT) in oligometastatic NSCLC patients with up to five metastatic lesions and negative oncogene mutations.

\section{MATERIALS AND METHODS}

This study is designed as a single institution, open-label, phase-III RCT, approved by the institutional ethics committee-II (IEC-II) (project number 3445). The study schema is shown in figure 1. All NSCLC patients with up to five metastatic sites at presentation will be screened for this study. Patients who have completed standard systemic therapy and response imaging shows no progressive disease (PD) as per Response Evaluation Criteria in Solid Tumors (RECIST) version 1.1 will be eligible for this study. If found eligible under eligibility criteria (table 1), patients will be explained about the study protocol by the study investigators in their native language and interested patients will be given an IEC approved written informed consent document available in English, Hindi and Marathi language (online supplemental file 1).

Patients will be randomised in a 1:1 ratio to SMT alone (control arm), and LCRT+SMT (experimental arm). Patients will be stratified by the number of metastatic sites (1-2 vs 3-5), nodal metastases (N0-N1 vs N2) and brain metastases (present vs absent). Independent biostatistician of the institute will perform a computer-generated randomisation sequence based on stratification factors. The study is expected to start from January 2021 and will continue for at least 5 years thereafter.

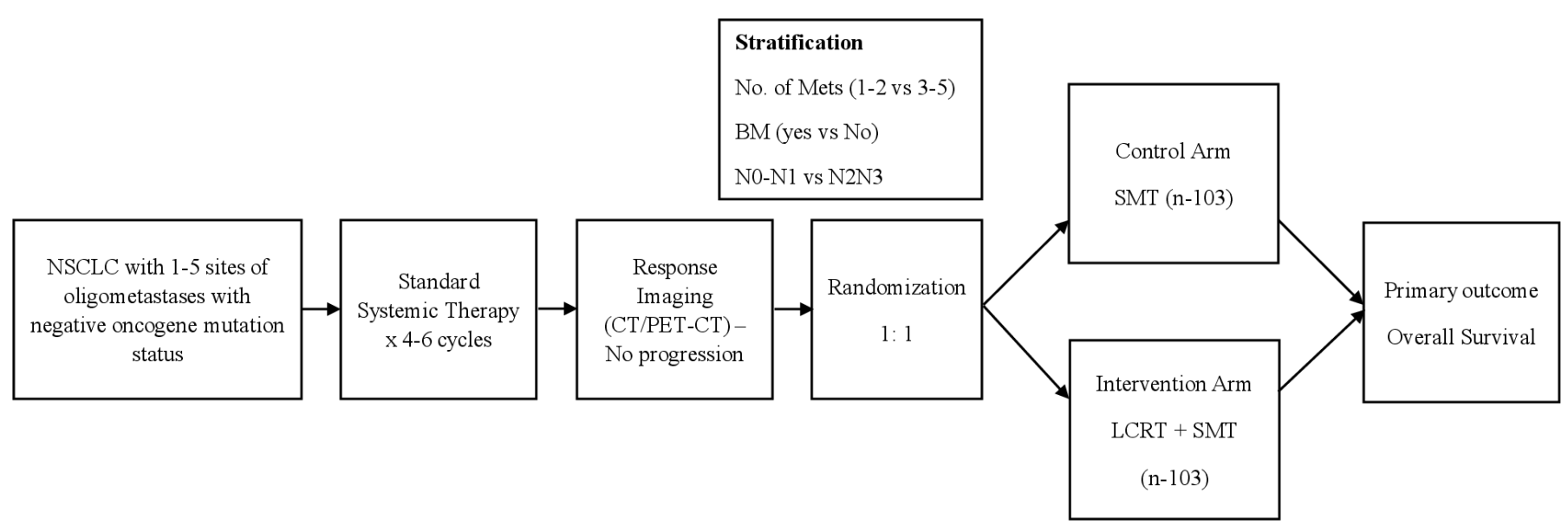

Figure 1 Study schema. BM, Brain metastases; CT, Computed Tomography; LCRT, local consolidative radiation therapy; NSCLC, non-small cell lung cancer; PET, positron emission tomography; SMT, standard maintenance therapy. 
Table 1 Eligibility criteria for the study

\begin{tabular}{|c|c|}
\hline Inclusion criteria & Exclusion criteria \\
\hline $\begin{array}{l}\text { 1. Age }>18 \text { years. } \\
\text { 2. ECOG performance status of } 0-2 \text {. } \\
\text { 3. Pathologically proven diagnosis of NSCLC. } \\
\text { 4. } 1-5 \text { sites of metastatic disease not including the primary tumour and } \\
\text { regional nodes ( } \leq 3 \text { metastatic lesions in one organ will be eligible). } \\
\text { 5. Patients should have received at least } 4-6 \text { cycles of systemic therapy } \\
\text { without progression on response imaging. } \\
\text { 6. Patients suitable for definitive therapy to the primary disease. } \\
\text { 7. All the oligometastatic lesions should be radiologically visible and suitable } \\
\text { for SBRT. } \\
\text { 8. Adequate end organ function with } \mathrm{CBC} / \text { differential obtained within } 15 \\
\text { days before registration on the study, with adequate bone marrow function } \\
\text { defined as follows: } \\
\text { a. Absolute neutrophil count } \geq 500 \text { cells } / \mathrm{mm}^{3} \text {. } \\
\text { b. Platelets } \geq 50 \times 10^{9} \text { cells } / \mathrm{mm}^{3} \text {. } \\
\text { c. Haemoglobin } \geq 80 \mathrm{~g} / \mathrm{L} \text { (use of transfusion or other intervention to achieve } \\
\quad \text { Hgb } \geq 8.0 \mathrm{~g} / \mathrm{dL} \text { is acceptable). } \\
\text { 9. Negative serum or urine pregnancy test for females of childbearing } \\
\text { potential, within } 14 \text { days before study registration. } \\
\text { 10. Patients willing for written informed consent and must be willing to comply } \\
\text { with the specified follow-up schedule. }\end{array}$ & $\begin{array}{l}\text { 1. Progressive disease after initial systemic therapy. } \\
\text { 2. Positive oncogene mutations (EGFR/ALK/ROS). } \\
\text { 3. More than } 5 \text { sites of oligo metastases. } \\
\text { 4. Metastatic lesion size }>5 \mathrm{~cm} \text {. } \\
\text { 5. More than three metastatic lesions in one organ. } \\
\text { 6. Malignant peritoneal disease. } \\
\text { 7. Malignant pleural effusion. } \\
\text { 8. Leptomeningeal disease. } \\
\text { 9. Brain metastases in the brain stem. } \\
\text { 10. Clinical or radiological evidence of spinal cord compression or metastases } \\
\text { within } 2 \mathrm{~mm} \text { of the spinal cord on MRI. } \\
\text { 11. Severe, active comorbidity defined as follows: } \\
\text { - Unstable angina and/or congestive heart failure requiring hospitalisation } \\
\text { - Transmural myocardial infarction within the last } 6 \text { months. } \\
\text { - Chronic obstructive pulmonary disease exacerbation or other respiratory } \\
\text { illness requiring hospitalisation or precluding study therapy at the time of } \\
\text { 12. History of radiation therapy to the thorax. } \\
\text { 13. History of malignancy within the last } 3 \text { years. }\end{array}$ \\
\hline
\end{tabular}

ALK, anaplastic lymphoma kinase; CBC, Complete Blood Count; ECOG, Eastern Cooperative Oncology Group; EGFR, epidermal growth factor receptor; NSCLC, non-small cell lung cancer; ROS, c-ros proto-oncogene 1 ; SBRT, stereotactic body radiation therapy.

\section{Patient and public involvement}

Patients or the public were not involved in the design, conduct, reporting or dissemination plans of this study.

\section{Objectives and endpoints}

The primary objective of this study is to assess the efficacy of LCRT after initial systemic therapy in oligometastatic NSCLC patients.

\section{Primary endpoint}

To compare the OS between SMT arm and LCRT+SMT arm where OS is defined as the time from the date of randomisation to the date of death due to any cause.

\section{Secondary endpoints}

1. PFS: the time from the date of randomisation until the date of disease progression, or until death in the absence of progression, whichever is earlier.

2. Local control for sites treated with LCRT: defined as the absence of PD (complete response (CR), partial response (PR) or stable disease).

3. New distant metastases free survival (DMFS): the time from the date of randomisation until the emergence of new distant metastases or death, whichever is earlier.

4. Objective response rate $(\mathrm{CR}+\mathrm{PR})$.

5. Patient-reported outcomes using the EORTC Quality Of Life (QOL) core questionnaire (QLQ-C30) and the corresponding lung cancer module (QLQ-LC13).

6. Treatment-related toxicity assessed using National Cancer Institute Common Toxicity Criteria version 5.

\section{Exploratory endpoints}

1. Textural features of primary and metastatic sites using the TexRAD software (TexRAD, Cambridge, UK).

2. Differences in the textural features between pretreatment and post-treatment images in the experimental arm and their correlation with survival outcomes.
3. Correlation of circulating tumour cells (CTCs) with the survival outcomes.

\section{Specific scenarios for inclusion}

1. Patients who underwent ablative radiation therapy (RT) or surgery or radiofrequency ablation (RFA) for metastatic sites at presentation or during systemic therapy will be eligible provided the site is under control and the total number of oligometastatic sites at the time of study entry (treated site included) is $\leq 5$.

2. Palliative RT for symptomatic bony metastases will be eligible provided the treated site is under control and further ablative doses of radiation can be delivered.

3. Patients with vertebral metastases who underwent surgical decompression, or stabilisation followed by palliative RT will be eligible in the study provided the treated site is under control and the patient has $\leq 5$ sites (treated site included).

\section{Prerandomisation assessment}

Eligible patients will undergo response assessment Positron Emission Tomography-Computed Tomography (PET-CT) or contrast-enhanced CT of thorax, abdomen, and pelvis after the completion of 4-6 cycles of standard systemic therapy. Complete history and thorough physical examination including performance status (PS) assessment, baseline laboratory tests (including but not limited to complete blood count, renal function tests and liver function tests), two-dimensional echocardiography and gadolinium contrast-enhanced MR brain if not done earlier. Patients who do not have a PD as per RECIST version 1.1 will be eligible.

\section{Defining the number of oligometastases}

All metastatic sites at presentation and on follow-up imaging will be confirmed by an experienced radiologist 
and will be discussed in multidisciplinary joint clinics. The involvement of adjacent vertebrae by direct extension would be counted as one site and not two sites of metastases. Indeterminate parenchymal lung nodule or any suspicious lung lesion on baseline imaging will be again evaluated on the response imaging for its metastatic confirmation. If required, biopsy confirmation will be preferred but is not mandatory. Primary tumour and regional nodes' feasibility for definitive RT will be assessed by the study investigators before randomisation. Non-regional nodes will be counted as an individual metastatic site. Patients who have received palliative RT for symptomatic bone and brain metastases during the initial period will be evaluated for local control of those sites. Additional ablative doses will be decided as per the study investigators' discretion.

\section{Control arm-SMT}

All patients in this arm will receive SMT which includes CT, immunotherapy or observation. SMT will be decided by the treating medical oncologist. Maintenance systemic therapy should start within 4-8 weeks of randomisation. Palliative RT to existing metastatic sites in this arm will be done on clinical or radiological worsening. Acceptable RT doses include $8 \mathrm{~Gy}$ times one or two fractions or $20 \mathrm{~Gy}$ in five fractions. No ablative doses to metastatic sites are allowed in this arm.

\section{Experimental arm-LCRT+SMT}

Patients in this arm will receive LCRT with SBRT to all oligometastatic sites and definitive RT to primary disease including involved regional nodes. SMT will be given as discussed in arm A. LCRT will be started within 4 weeks of randomisation. Maintenance systemic therapy can be started concurrently or after completion of LCRT within 4 weeks. SBRT doses are given in table 2. Definitive radiation for the primary and nodal disease would be done similarly as in locally advanced NSCLC with hypofractionation schedule to a dose of 45-55 Gy in 15-22 fractions. Doses will be decided depending on normal tissue tolerances and at radiation oncologist's discretion.

\section{Radiotherapy planning and delivery}

All patients in the experimental arm will undergo CT-based planning which should completely cover all the areas of interest so that a composite dose distribution can be created for all metastatic sites. Ideally, patient position will be preferred to remain the same across all treatment sites except brain metastases. Four-dimensional CT will be used to encompass tumour and organ motion for moving metastatic sites. Target volume delineation will be done as per the consensus guidelines for individual sites, for example, international spinal consortium guidelines for spinal metastases. ${ }^{24} \mathrm{~A}$ high precision technique like SBRT will be used for oligometastatic sites. Treatment planning will be done using intensity-modulated RT or volumetric modulated arc therapy.

\section{Radiomics}

Texture analysis of medical images like CT and MRI assess heterogeneity of tumours and other benign lesions. ${ }^{2526} \mathrm{It}$ evaluates the distribution of grey levels, coarseness and regularity. As a radiomics endpoint, texture analysis will be done on pretreatmentand post-treatment imaging and their significance and correlation will be analysed separately.

\section{Circulating tumour cells}

In this study, the exploratory translational objective is to evaluate the significance of CTCs in the blood at baseline and subsequent follow-up. CTCs have been identified as a prognostic marker in different tumour subtypes. ${ }^{27} 28$ Serial follow-up of CTCs in blood could predict clinical recurrence earlier than the radiological recurrence.

\section{Participant withdrawal/discontinuation}

The principal investigator can discontinue the treatment whenever deemed necessary if the patient has significant toxicities or in life-threatening clinical scenarios. Patients can withdraw from the study without giving any reasons, however, reasons for withdrawal would be preferred for study documentation. Any data prior to withdrawal will be used for the study related outcome analysis.

\section{Safety monitoring}

The data safety monitoring committee of the institute will monitor the progress of the study at regular intervals. Study modifications/amendments will be informed to Institutional Review Board (IRB) for approval, study sponsors, and will be uploaded in the Clinical Trials

\begin{tabular}{|c|c|c|c|c|c|}
\hline Oligometastatic site & Location & Dose per fraction (Gy) & Fractions (n) & Total dose & Frequency \\
\hline $\begin{array}{l}\text { Primary (if NO) and } \\
\text { lung metastases }\end{array}$ & $\begin{array}{l}\text { Peripheral } \\
\text { Central } \\
\text { Ultra-central }\end{array}$ & $\begin{array}{l}12 \\
7.5 \\
5\end{array}$ & $\begin{array}{l}5 \\
8 \\
10\end{array}$ & $\begin{array}{l}60 \text { Gy } \\
60 \text { Gy } \\
50 \text { Gy }\end{array}$ & $\begin{array}{l}\text { Alternate day } \\
\text { Daily/alternate } \\
\text { Daily/alternate }\end{array}$ \\
\hline Bone & $\begin{array}{l}\text { Spine } \\
\text { Any other }\end{array}$ & $\begin{array}{l}8-12 \\
7\end{array}$ & $\begin{array}{l}3-2 \\
5\end{array}$ & $\begin{array}{l}24 \mathrm{~Gy} \\
35 \mathrm{~Gy}\end{array}$ & $\begin{array}{l}\text { Alternate day } \\
\text { Daily/alternate }\end{array}$ \\
\hline Brain & $\begin{array}{l}\text { Single } \\
1-3 \text { lesion }\end{array}$ & $\begin{array}{l}18-24 \\
18-24 \text { or } 5\end{array}$ & $\begin{array}{l}1 \\
1 \text { or } 10\end{array}$ & $\begin{array}{l}18-24 \\
18-24 \text { or } 50\end{array}$ & $\begin{array}{l}\text { Single } \\
\text { Single/daily }\end{array}$ \\
\hline Adrenal & NA & 7-10Gy & 5 & 35-50Gy & Daily/alternate \\
\hline Liver & Any & 6-10Gy & 5 & 30-50Gy & Daily/alternate \\
\hline
\end{tabular}


Registry-India. All toxicities, treatment interruptions or discontinuation and protocol deviations will be recorded and inform by the study investigators to the institutional review board as specified by the institutional guidelines.

\section{Statistics}

\section{Randomisation}

All eligible patients will be stratified according to the number of metastatic sites (1-2 vs 3-5), nodal status (N0-N1 vs N2-N3) and brain metastases (present vs absent). Patients will then undergo 1:1 randomisation by an independent biostatistician with permuted block randomisation.

\section{Sample size calculation}

The results of the phase II study of Gomez et al demonstrated a median OS of 17 months in the SMT alone arm and 41.2 months in SMT+LCTarm. For this phase III study, we took median OS of 17 months in the standard arm from Gomez et al and are expecting an increment of 10 months in the experimental arm with HR of 0.63 based on previous single arm phase II studies. ${ }^{29} 30$ To detect this difference, with $80 \%$ power and a two-sided alpha of 0.05 , 148 events will be required, 80 in the control arm and 68 in the experimental arm. Assuming a 10\% drop out rate, the total sample size required would be 206 (103 in the control arm and 103 in the experimental arm). We intend to accrue 40-45 patients per year for a 5-year accrual period with a minimum follow-up of 2 years. The total study duration is 7 years.

\section{Analysis}

Study-related data will be collected in an electronic case record form and will be uploaded in a restricted-access database (REDCap). Data will be available to principal investigators and the statistical team of the study in a password protected computer folder. Patient baseline characteristics will be summarised by study arm and control arm. $\chi^{2}$ test or Fisher's exact test will be applied to compare patient characteristics between the two arms. The primary endpoint of OS will be calculated as per the intentionto-treat analysis. OS, PFS and DMFS will be calculated using the Kaplan-Meier method and log-rank test will be used for comparison between the groups. Cox proportional hazard regression model will be used to analyse the effects of factors, in addition to treatment, that may be associated with OS and PFS. $\chi^{2}$ test with Pearson's or Fisher's exact test will be used to compare the objective response rates (ORR) between the two treatment arms. A similar test will be used to estimate the incidence of adverse events in each treatment arm (both acute and chronic toxicities). A p value $\leq 0.05$ in a two-tailed test will be considered statistically significant. Statistical analyses will be performed using SPSS V.25.0 (Statistical Package for Social Sciences) and STATA V.14.

\section{Quality Of Life (QOL) analysis}

The EORTC QLQ-LC13 is a 13-item questionnaire grouped, while the QLQ-C30 comprises a 30-item questionnaire. Raw scores will be standardised by linear transformation such that the final scores ranged between 0 and 100. Higher scores on the global QOL and functional scales represent a better QOL, whereas high symptom scale scores indicate significant symptoms or greater difficulty. If data from one assessment point will be missing, then the last observation carry forward method will be used to impute the missing subsequent values. To account for possible bias due to imputation, sensitivity analyses will be performed by conducting a complete case analysis. Repeated-measures analysis of variance will be used to assess the interaction of time and group with time as within-subject factor and group as a between-subject factor with respect to EORTC QLQ 30 and LC30 from baseline to till the last follow-up.

\section{Follow-up evaluation and toxicity assessment}

All patients accrued in the control arm will be followed up every 3 monthly ( \pm 4 weeks) for the first 2 years and then 6 monthly $( \pm 6$ weeks) afterward till 5 years and thereafter annually (table 3). Patients in the experimental arm during LCRT treatment will be reviewed once weekly for symptom and acute toxicity assessment if any. Post-LCRT completion follows up will be done similarly

Table 3 Follow-up visits schedule

\begin{tabular}{|c|c|c|c|c|}
\hline Assessment & Before randomisation & $\begin{array}{l}\text { First follow up at } 3 \\
\text { months ( } \pm 4 \text { weeks) }\end{array}$ & $\begin{array}{l}\text { Every } 3 \text { months till } 2 \\
\text { years ( } \pm 4 \text { weeks) }\end{array}$ & $\begin{array}{l}\text { Six monthly till } 5 \\
\text { years ( } \pm 6 \text { weeks) }\end{array}$ \\
\hline Physical examination & $x$ & $x$ & $x$ & $x$ \\
\hline $\mathrm{RO}$ assessment & $x$ & $x$ & $x$ & $x$ \\
\hline $\mathrm{CECT}(\mathrm{T}+\mathrm{A}+\mathrm{P})$ & $x$ & $x$ & $x$ & $x$ \\
\hline PET CT & Not required (preferred) & Not required & As required & As required \\
\hline QOL questionnaires & $x$ & $x$ & $x$ (at 6 and 12 months) & - \\
\hline
\end{tabular}

CECT, Contrast enhanced Computed Tomography; MRI, Magnetic Resonance Imaging; PET, Positron Emission Tomography; QOL, Quality Of Life; RO, Radiation Oncology. 


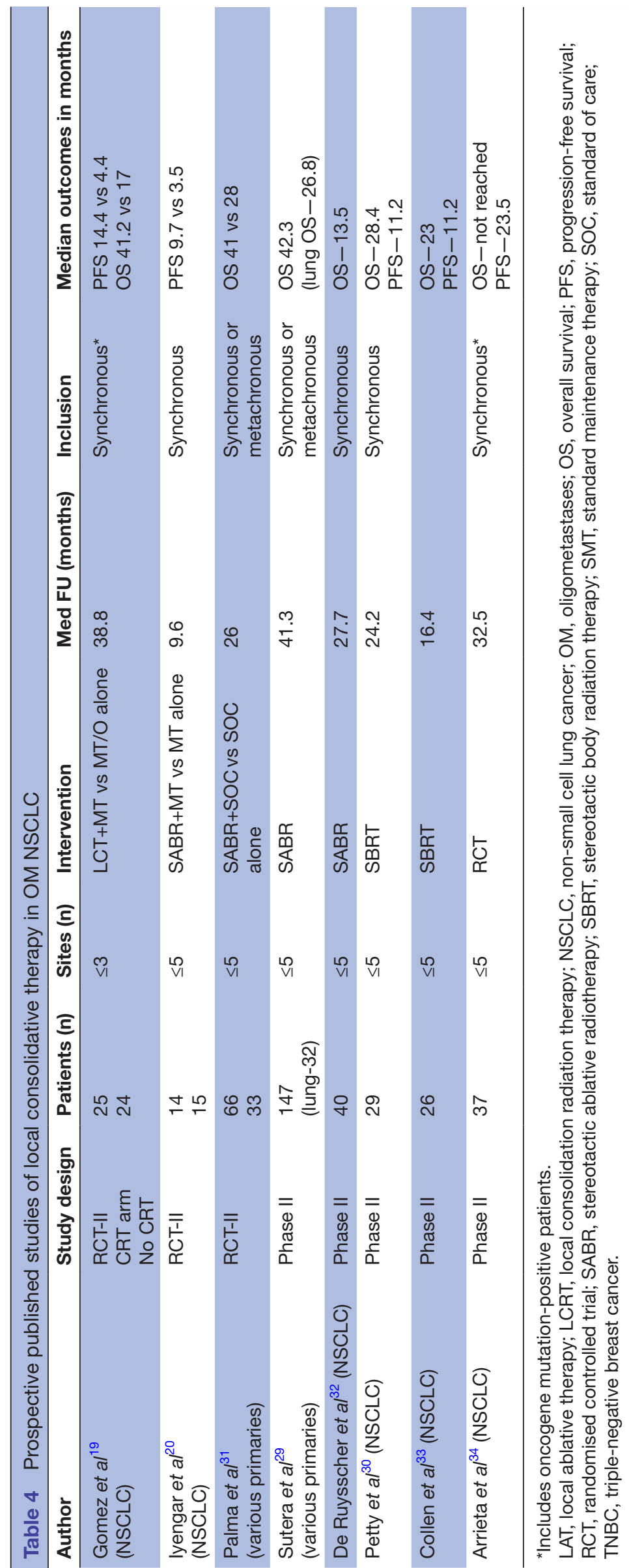

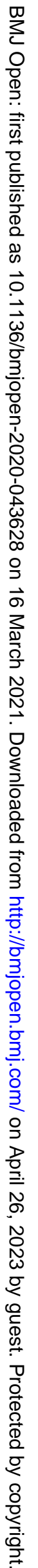




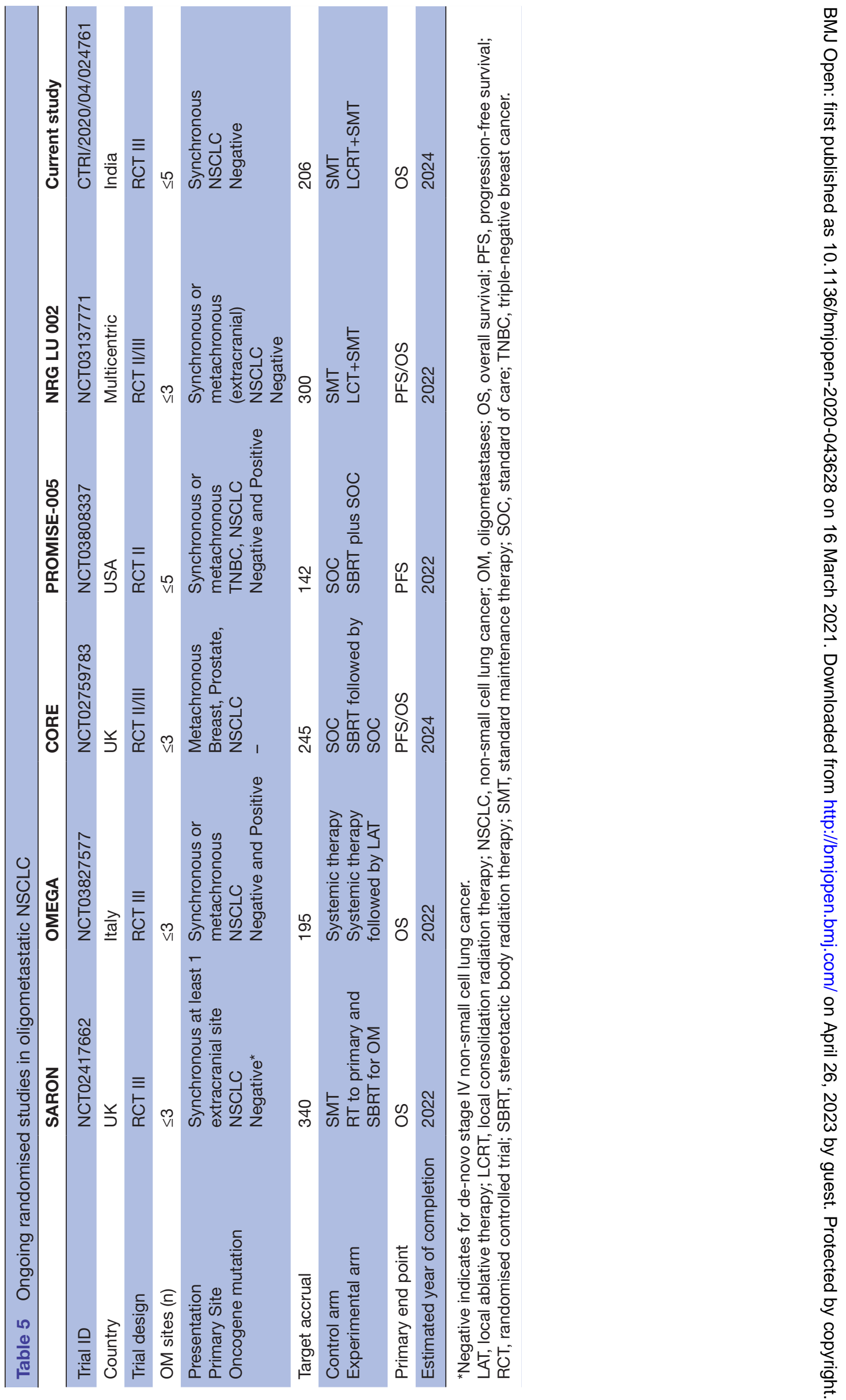


as in the control arm. Adherence to protocol treatment and timely follow-up of participants will be encouraged by the study investigators and team members through proper counselling, resolving queries and by allowing easy access to them. Any serious adverse events during treatment and at follow-up will be documented, informed to IRB, managed appropriately and will be followed up till resolution. At each visit, history and physical examination, Eastern Cooperative Oncology Group (ECOG) PS, toxicity assessment using NCI CTC version 5.0 will be recorded. Acute toxicity is defined as symptoms occurring within 90 days of the first fraction of radiotherapy. Late toxicity is defined as symptoms occurring beyond 90 days. Efficacy assessment will be done with CT imaging of the disease sites at every follow-up. On equivocal suspicion of recurrent or PD, PET-CT or biopsy will be done. If biopsy not feasible, repeat imaging will be done after 4-8 weeks. MRI brain will be done on clinical suspicion based on neurological worsening. EORTC QOL questionnaires will be completed by patients at 3,6 and 12 months. If symptomatic before the scheduled follow-up visit, relevant imaging will be done to rule out progression.

\section{Treatment at the progression}

Isolated progression at existing or new sites will be evaluated for local ablative therapies including surgery, SBRT or RFA in both the arms. For limited or widespread metastases, subsequent lines of CT or immunotherapy will be decided at the investigator's discretion depending on PS at the time of progression. Symptomatic sites will be offered palliative RT as per the existing institutional policies. Follow-up schedule after progression will be adjusted to match the existing schedules within \pm 6 weeks to avoid duplication of visits.

\section{Quality assurance}

Strict adherence to quality assurance protocols will be ensured for patients undergoing SBRT or definitive RT in the experimental arm. Full quality assurance guidelines will be published separately.

\section{Ethics and dissemination}

The study is approved by the institutional ethics committee of Tata Memorial Hospital (TMH IRB project number 3445). The study is registered prospectively with Clinical Trials Registry-India, dated 21 April 2020. Written informed consent will be obtained from all the patients for study interventions, biomarkers and radiomics part of the study.

\section{Confidentiality}

Study participants' names and personal information will be held in strict confidence and will not be shared publicly. Participant details in case record forms, safety reports and correspondence to IRB will be done with the study identification number and participant's initials. Study investigators will maintain a master list with the participant's identification details.

\section{DISCUSSION}

Oligometastatic disease deserves attention owing to the increasing evidence from various retrospective and prospectively randomised studies. Two-phase II randomised studies in NSCLC patients have shown significant benefit in PFS (table 4). Adequately powered wellconducted phase III RCT is needed to generate level I evidence to support the efficacy of local ablative therapies in OM NSCLC. There are two similar phase III RCT in progress for assessment of local ablative therapy in combination with systemic therapy (table 5).

The SARON trial (NCT02417662) is a multicentere, randomised phase III trial being conducted in 30 hospitals in the UK and plans to recruit 340 patients with oligometastatic EGFR, ALK and ROS1 mutation-negative NSCLC (1-3 sites of synchronous metastatic disease at least one of which must be extracranial). Patients will receive either standard systemic therapy only or standard systemic therapy plus radical radiotherapy or SBRT to their primary tumours (and mediastinal nodes where present) and SBRT/Stereotactic radiosurgery to all metastatic sites. The primary end-point of the study is OS.

The OMEGA trial (NCT03827577) is a phase III randomised trial being conducted in Italy which proposes to recruit 195 patients with synchronous or metachronous oligometastatic NSCLC with up to three metastatic sites. The study will include both oncogene mutation-positive and negative patients and they will be randomised to receive either standard systemic therapy alone (platinum doublet CT or TKI or immunotherapy) or standard systemic therapy followed by SBRT, surgical resection or RFA. The primary endpoint of the study is OS.

Our institute sees approximately 2500 new lung cancer patients annually. The proposed study is a single-centre study and will recruit patients with $\leq 5 \mathrm{OM}$ sites after completion of initial planned standard systemic therapy. This study will also include patients who have been treated with palliative RT at presentation and if controlled at the time of randomisation. The possibility of further ablative doses at those particular sites will be ascertained by the radiation oncologist. The study is currently awaiting funding from extramural grants and will start recruitment once funding is arranged.

Author affiliations
'Department of Radiation Oncology, Tata Memorial Hospital, Homi Bhabha National
Institute, Mumbai, India
2Department of Medical Oncology, Tata Memorial Hospital, Homi Bhabha National
Institute, Mumbai, India
${ }^{3}$ Department of Nuclear Medicine, Tata Memorial Hospital, Homi Bhabha National
Institute, Mumbai, India
${ }^{4}$ Department of Radiodiagnosis, Tata Memorial Hospital, Homi Bhabha National
Institute, Mumbai, India
${ }^{5}$ Clinical Research Secretariat, Tata Memorial Hospital, Homi Bhabha National
Institute, Mumbai, India

Twitter Anil Tibdewal @ANILTIBDEWAL and Vanita Noronha @VanitaNoronha Contributors Concept and design of the study: AT and JPA. The initial draft of protocol: AT, JPA. The final draft of the protocol: AT, JPA, SS, NM, KP, VN, VP, NP, AJ and SK. All authors read and approved the final protocol. 
Funding The authors have not declared a specific grant for this research from any funding agency in the public, commercial or not-for-profit sectors.

Competing interests VN has received institutional research funding from Amgen, Sanofi India, Dr. Reddy's Laboratories, Intas Pharmaceuticals and Astra Zeneca Pharma India. All research grants have been paid to the institution. KP has received research funding from Dr. Reddy's Laboratories, Fresenius Kabi India, Alkem Laboratories, Natco Pharma, BDR Pharmaceuticals Intl. and Roche Holding AG (all research grants paid to the institution and are unrelated to this study project). The rest of the authors declare that they have no competing interest in the proposed study protocol.

Patient consent for publication Not required.

Provenance and peer review Not commissioned; externally peer reviewed.

Supplemental material This content has been supplied by the author(s). It has not been vetted by BMJ Publishing Group Limited (BMJ) and may not have been peer-reviewed. Any opinions or recommendations discussed are solely those of the author(s) and are not endorsed by BMJ. BMJ disclaims all liability and responsibility arising from any reliance placed on the content. Where the content includes any translated material, BMJ does not warrant the accuracy and reliability of the translations (including but not limited to local regulations, clinical guidelines, terminology, drug names and drug dosages), and is not responsible for any error and/or omissions arising from translation and adaptation or otherwise.

Open access This is an open access article distributed in accordance with the Creative Commons Attribution Non Commercial (CC BY-NC 4.0) license, which permits others to distribute, remix, adapt, build upon this work non-commercially, and license their derivative works on different terms, provided the original work is properly cited, appropriate credit is given, any changes made indicated, and the use is non-commercial. See: http://creativecommons.org/licenses/by-nc/4.0/.

ORCID iD

Anil Tibdewal http://orcid.org/0000-0002-0374-0800

\section{REFERENCES}

1 Solomon BJ, Mok T, Kim D-W, et al. First-Line crizotinib versus chemotherapy in ALK-positive lung cancer. $N$ Engl J Med 2014:371:2167-77.

2 Soria J-C, Ohe Y, Vansteenkiste J, et al. Osimertinib in untreated EGFR-mutated advanced non-small-cell lung cancer. N Engl J Med 2018;378:113-25.

3 Rosell R, Carcereny E, Gervais R, et al. Erlotinib versus standard chemotherapy as first-line treatment for European patients with advanced EGFR mutation-positive non-small-cell lung cancer (EURTAC): a multicentre, open-label, randomised phase 3 trial. Lancet Oncol 2012;13:239-46.

4 Patil VM, Noronha V, Joshi A, et al. Phase III study of gefitinib or pemetrexed with carboplatin in EGFR-mutated advanced lung adenocarcinoma. ESMO Open 2017;2:e000168-8.

5 Reck M, Rodríguez-Abreu D, Robinson AG, et al. Pembrolizumab versus chemotherapy for PD-L1-positive non-small-cell lung cancer. N Engl J Med 2016;375:1823-33.

6 Mok TSK, Wu Y-L, Kudaba I, et al. Pembrolizumab versus chemotherapy for previously untreated, PD-L1-expressing, locally advanced or metastatic non-small-cell lung cancer (KEYNOTE-042): a randomised, open-label, controlled, phase 3 trial. Lancet 2019;393:1819-30.

7 Ashworth AB, Senan S, Palma DA, et al. An individual patient data metaanalysis of outcomes and prognostic factors after treatment of oligometastatic non-small-cell lung cancer. Clin Lung Cancer 2014;15:346-55.

8 Van den Begin R, Engels B, Collen C, et al. The METABANK score: a clinical tool to predict survival after stereotactic radiotherapy for oligometastatic disease. Radiother Oncol 2019;133:113-9.

9 Hellman S, Weichselbaum RR. Oligometastases. J Clin Oncol 1995;13:8-10.

10 Weichselbaum RR, Hellman S. Oligometastases revisited. Nat Rev Clin Oncol 2011;8:378-82.

11 Gomez DR, Tang C, Zhang J, et al. Local consolidative therapy vs. maintenance therapy or observation for patients with oligometastatic non-small-cell lung cancer: long-term results of a multi-institutional, phase II, randomized study. J Clin Oncol 2019;37:1558-65.

12 Dingemans A-MC, Hendriks LEL, Berghmans T, et al. Definition of synchronous oligometastatic non-small cell lung cancer-a consensus report. J Thorac Oncol 2019;14:2109-19.
13 Lievens Y, Guckenberger M, Gomez D, et al. Defining oligometastatic disease from a radiation oncology perspective: an ESTRO-ASTRO consensus document. Radiother Oncol 2020;148:157-66.

14 Rusthoven KE, Hammerman SF, Kavanagh BD, et al. Is there a role for consolidative stereotactic body radiation therapy following first-line systemic therapy for metastatic lung cancer? A patterns-offailure analysis. Acta Oncol 2009;48:578-83.

15 Patel SH, Rimner A, Foster A, et al. Patterns of initial and intracranial failure in metastatic EGFR-mutant non-small cell lung cancer treated with erlotinib. Lung Cancer 2017;108:109-14.

16 Al-Halabi H, Sayegh K, Digamurthy SR, et al. Pattern of failure analysis in metastatic EGFR-mutant lung cancer treated with tyrosine kinase inhibitors to identify candidates for consolidation stereotactic body radiation therapy. J Thorac Oncol 2015;10:1601-7.

17 Parikh RB, Cronin AM, Kozono DE, et al. Definitive primary therapy in patients presenting with oligometastatic non-small cell lung cancer. Int J Radiat Oncol Biol Phys 2014;89:880-7.

18 Xu Q, Wang Y, Liu H, et al. Treatment outcome for patients with primary NSCLC and synchronous solitary metastasis. Clin Trans/ Oncol 2013;15:802-9.

19 Gomez DR, Blumenschein GR, Lee JJ, et al. Local consolidative therapy versus maintenance therapy or observation for patients with oligometastatic non-small-cell lung cancer without progression after first-line systemic therapy: a multicentre, randomised, controlled, phase 2 study. Lancet Oncol 2016;17:1672-82.

20 lyengar P, Wardak Z, Gerber DE. Consolidative radiotherapy for limited metastatic non-small-cell lung cancer: a phase 2 randomized clinical trial. JAMA Oncol 2018;4:1-4.

21 Elamin YY, Gomez DR, Antonoff MB, et al. Local consolidation therapy (Ict) after first line tyrosine kinase inhibitor (TKI) for patients with EGFR mutant metastatic non-small-cell lung cancer (NSCLC). Clin Lung Cancer 2019;20:43-7.

22 Wang $X$, Zeng M. First-Line tyrosine kinase inhibitor with or without aggressive upfront local radiation therapy in patients with EGFRm oligometastatic non-small cell lung cancer: interim results of a randomized phase III, open-label clinical trial (SINDAS) (NCT02893332). JCO 2020;38:9508.

23 Mak RH, Doran E, Muzikansky A, et al. Outcomes after combined modality therapy for EGFR-mutant and wild-type locally advanced NSCLC. Oncologist 2011;16:886-95.

24 Cox BW, Spratt DE, Lovelock M, et al. International spine radiosurgery Consortium consensus guidelines for target volume definition in spinal stereotactic radiosurgery. Int J Radiat Oncol Biol Phys 2012;83:e597-605.

25 Dou TH, Coroller TP, van Griethuysen JJM, et al. Peritumoral radiomics features predict distant metastasis in locally advanced NSCLC. PLoS One 2018;13:e0206108-15.

26 Aerts HJWL, Velazquez ER, Leijenaar RTH, et al. Decoding tumour phenotype by noninvasive imaging using a quantitative radiomics approach. Nat Commun 2014;5:4006.

27 Alix-Panabières C, Pantel K. Clinical applications of circulating tumor cells and circulating tumor DNA as liquid biopsy. Cancer Discov 2016;6:479-91.

28 Lindsay CR, Faugeroux V, Michiels S, et al. A prospective examination of circulating tumor cell profiles in non-small-cell lung cancer molecular subgroups. Ann Oncol 2017;28:1523-31.

29 Sutera P, Clump DA, Kalash R, et al. Initial results of a multicenter phase 2 trial of stereotactic ablative radiation therapy for oligometastatic cancer. Int J Radiat Oncol Biol Phys 2019;103:116-22.

30 Petty WJ, Urbanic JJ, Ahmed T, et al. Long-Term outcomes of a phase 2 trial of chemotherapy with consolidative radiation therapy for oligometastatic non-small cell lung cancer. Int J Radiat Oncol Biol Phys 2018;102:527-35.

31 Palma DA, Olson R, Harrow S, et al. Stereotactic ablative radiotherapy versus standard of care palliative treatment in patients with oligometastatic cancers (SABR-COMET): a randomised, phase 2, open-label trial. Lancet 2019;393:2051-8.

32 De Ruysscher D, Wanders R, Hendriks LE, et al. Progression-Free survival and overall survival beyond 5 years of NSCLC patients with synchronous Oligometastases treated in a prospective phase II trial (NCT 01282450). J Thorac Oncol 2018;13:1958-61.

33 Collen C, Christian N, Schallier D, et al. Phase II study of stereotactic body radiotherapy to primary tumor and metastatic locations in oligometastatic nonsmall-cell lung cancer patients. Ann Oncol 2014;25:1954-9.

34 Arrieta O, Barrón F, Maldonado F, et al. Radical consolidative treatment provides a clinical benefit and long-term survival in patients with synchronous oligometastatic non-small cell lung cancer: a phase II study. Lung Cancer 2019;130:67-75. 